\title{
Reduced glomerular filtration rate and prior cardiovascular event entail similar risk for coronary atherosclerotic burden
}

\section{Pamela Angela Piscitelli}

IRCCS Casa Sollievo della Sofferenza, San Giovanni Rotondo

Antonio Mangiacotti

IRCCS Casa Sollievo della Sofferenza, San Giovanni Rotondo

Nicola Marchese

IRCCS Casa Sollievo della Sofferenza, San Giovanni Rotondo

\section{Eulalia Valentina Greco}

IRCCS Casa Sollievo della Sofferenza, San Giovanni Rotondo

Maria Maddalena D'Errico

IRCCS Casa Sollievo della Sofferenza, San Giovanni Rotondo

Valentina Massa

IRCCS Casa Sollievo della Sofferenza, San Giovanni Rotondo

Antonio Mirijello

IRCCS Casa Sollievo della Sofferenza, San Giovanni Rotondo

\section{Antonio Pio Palena}

IRCCS Casa Sollievo della Sofferenza, San Giovanni Rotondo

Gianluigi Vendemiale

Department of Medical Sciences, University of Foggia

\section{Aldo Russo}

IRCCS Casa Sollievo della Sofferenza, San Giovanni Rotondo

\section{Carlo Vigna}

IRCCS Casa Sollievo della Sofferenza, San Giovanni Rotondo

\section{Roberto Pontremoli}

Università degli Studi and IRCCS Azienda Ospedaliera Universitaria San Martino-IST, Genova

Salvatore De Cosmo ( $\sim$ sdecosm@tin.it )

https://orcid.org/0000-0001-8787-8286

\section{Research article}

Keywords: Atherosclerosis, Chronic kidney disease, coronary angiography, previous CV event. 
Posted Date: August 26th, 2019

DOl: https://doi.org/10.21203/rs.2.13590/v1

License: (c) (i) This work is licensed under a Creative Commons Attribution 4.0 International License. Read Full License 


\section{Abstract}

Background: Prior cardiovascular event and kidney dysfunction are both strong risk factor for coronary artery disease. Aim of this study is to assess coronary atherosclerotic burden in a large population of patients undergoing coronary angiography, according to the prior cardiovascular event or chronic kidney disease. Methods: We evaluated 700 consecutive patients who underwent CA. Serum creatinine to estimate glomerular filtration rate (eGFR) was measured. Clinically significant CAD was defined by the presence of a coronary lesion resulting in a luminal stenosis $>50 \%$. For the purpose of the study, the whole population was divided into 4 subgroups according to the presence/absence of eGFR $<60$ $\mathrm{ml} / \mathrm{min} / 1.73 \mathrm{~m} 2$ or prior cardiovascular event: eGFR $\geq 60 /$ no event (Group A), eGFR $\geq 60 /$ yes event (Group B), eGFR $<60 /$ no event (Group C), eGFR $<60 /$ yes event (Group D). Results: As expected, patients in group D had the worst clinical and biochemical profile. These patients also showed the highest values of ACR $(p<0.001)$ and the lowest values of eGFR $(p<0.01)$. One-hundred-ninety-six patients had three vessel disease. Considering group $A$ as reference, the risk of having three-vessel disease was increased in group $\mathrm{B}(\mathrm{OR}=2.09 ; 95 \% \mathrm{Cl} 1.37-3.19)$, in group $\mathrm{C},(\mathrm{OR}=1.80 ; 95 \% \mathrm{Cl} 1.04-3.14)$ and finally in group $\mathrm{D}(\mathrm{OR}=3.35$; $95 \% \mathrm{Cl} 2.01-5.58)$. The risk carried by group $\mathrm{C}$ was not significantly different from that carried by Group $\mathrm{B}$ : $\mathrm{OR}=0.86 ; 95 \% \mathrm{Cl} 0.5-1.5$. Conclusions: In our study low eGFR seems to have the same excess risk of prior CV event.

\section{Background}

Cardiovascular disease is the leading cause of death worldwide as well as in the developed countries [1]. Atherosclerotic coronary artery disease (CAD) causes almost half of all cardiovascular deaths.

Mortality from coronary heart disease increases several times among patients with established coronary heart disease.[2]. Prior myocardial infarction significantly rises the risk of coronary and cardiovascular disease mortality [3].

Chronic kidney disease (CKD), which is diagnosed by the presence of persistent increased urine albumin excretion and/or decreased estimated glomerular filtration rate (eGFR) (i.e. eGFR<60 $\mathrm{ml} / \mathrm{min} / 1.73 \mathrm{~m} 2$ ), affects up to $17 \%$ of the general population in the European countries [4]. Decreased renal function is strongly associated with increased risk of cardiovascular events, including cardiovascular mortality [5-7]. The latter is the most frequent cause of death in patients with chronic kidney disease stage $\leq 3$ (i.e. GFR $<60 \mathrm{ml} / \mathrm{min} / 1.73 \mathrm{~m} 2$ ) and CAD is the most frequent cause of cardiovascular death in these patients [8]. Impaired eGFR and the presence of albuminuria associate with occurrence and severity of coronary atherosclerosis and, among patients with CAD, with major adverse cardiovascular outcome [9-10].

Taking into account these data, the American College of Cardiology/American Heart Association task force and the National Kidney Foundation in 2003 recommended that chronic kidney disease should be considered a coronary heart disease (CHD) risk equivalent [7]. 
However, whether kidney dysfunction carries a coronary atherosclerotic burden similar to that carried by patients with prior cardiovascular event is yet unknown.

Therefore, aim of our work was to assess coronary atherosclerotic burden in a large population of patients undergoing $\mathrm{CA}$, according to the prior cardiovascular event or chronic kidney disease.

\section{Methods}

Study cohort and baseline measurements.

In an ongoing observational study aiming to investigate the role of kidney dysfunction in modulating the risk of cardiovascular motility and mortality, we evaluated 700 consecutive patients who underwent coronary angiography (CA) from May 2016 to July 2017 at Coronary Unit of Scientific Institute "Casa Sollievo della Sofferenza". The study was approved by the local Ethical Committee. As already reported [10], all patients underwent diagnostic CA as part of the clinical work-up of their symptoms or signs of CAD. CA has been performed with Judkins technique in all patients. In the whole sample, 400 patients had a percutaneous coronary intervention ( $\mathrm{PCl}$ ) procedure. Each patient, in the morning of $\mathrm{CA}$, was interviewed for collecting demographic characteristics such as age, gender, height and weight, and smoking habits (no smoker, current smoker or ex-smoker). For each patient, we collected pathological and remote history, highlighting prior cardiovascular events, such as stroke, myocardial infarction, angina, prior myocardial revascularization, significant carotid stenosis, prior carotid revascularization, peripheral obliterative arteriopathy and prior peripheral revascularization. The same day all patients had blood pressure (BP) measured. Fasting venous blood was also sampled from an antecubital vein from all patients for the measurement of standardized serum creatinine by using the modified kinetic Jaffè reaction (Hitachi 737 Autoanalyzer), calibrated to be traceable to an isotope dilution mass spectrometry (IDMS), total serum cholesterol (enzymatic method, Cobas; Roche Diagnostics, Welwin Garden City, U.K.), HDL-cholesterol (HDL-c), serum triglycerides (enzymatic method, Cobas). Serum lipids including triglycerides, HDL-c and LDL-cholesterol (LDL-c) were measured by enzymatic methods.

Urinary albumin and creatinine concentration were determined by the nephelometric method (Behring Nephelometer Analyzer; Behring, Marburg, Germany) and the Jaffè reaction rate method, respectively. Urinary albumin excretion was calculated and reported as urinary albumin creatinine ratio (ACR). We defined: normoalbuminuria if $A C R \leq 2.5$ in men and $\leq 3.5 \mathrm{mg} / \mathrm{mmol}$ in women; microalbuminuria if $A C R$ was $>2.5 \mathrm{mg} / \mathrm{mmol}$ in men and $>3.5 \mathrm{mg} / \mathrm{mmol}$ in women and $\leq 30 \mathrm{mg} / \mathrm{mmol}$ in both gender; macroalbuminuria if ACR was $>30 \mathrm{mg} / \mathrm{mmol}$ in both gender. In the data analysis we have considered both microalbuminuria and macroalbuminuria as named albuminuria. GFR was estimated using the Chronic Kidney Disease Epidemiology Collaboration formula derived by standardized serum creatinine values. CKD was defined by the presence of albuminuria or eGFR $<60 \mathrm{ml} / \mathrm{min} / 1.73 \mathrm{~m} 2$ (i.e. low eGFR), or both. Patients were classified as having T2DM if they reported a prior diagnosis or were receiving antidiabetic treatment. Glycated haemoglobin (HbA1c) was measured by HPLC (Diamat Analyzer; BioRad, Richmond, $C A$ ) in patients affected by diabetes. Antihypertensive, lipid-lowering and 
antihyperglycemic treatments were also recorded. As already reported (10), clinically significant CAD was defined as the presence of a coronary lesion with a lumen diameter stenosis $\geq 50 \%$ in a major epicardial artery. Patients were then stratified according to the number of involved vessels as follows: single vessel disease (S VD), two vessels disease (2 VD) and three vessels disease (3 VD).

According to the purpose of the study, the whole examined population was divided into 4 subgroups according to the presence/absence of eGFR $<60 \mathrm{ml} / \mathrm{min} / 1.73 \mathrm{~m} 2$ or prior cardiovascular event: eGFR $\geq 60 /$ no event (Group A), eGFR $\geq 60 /$ yes event (Group B), eGFR $<60 /$ no event (Group C), eGFR $<60 /$ yes event (Group D).

\section{Statistical Analyses}

Continuous variables are expressed as mean \pm SD or median (range); categorical variables are described as frequencies and percentages. Mean differences were compared by unpaired Student's $t$ or 1-way Anova tests, as appropriate. Differences between categorical variables were tested by $\mathrm{X}^{2}$.

A multivariate logistic regression model was fitted to estimate the effect of kidney measures or prior CV event on the risk of having a three vessel disease. Two models of analysis were used. The first one, Group A, was the reference group for all others, in the second model (i.e. repeated analysis) the prior group was the reference one for each following group (i.e. group B vs. group A, group C vs group B and, finally, group D vs group C). The results, adjusted for age and gender, are expressed as odds ratio and their $95 \%$ confidence interval.

A p value less than 0.05 was considered to be significant.

All data analyses have been performed with the SPSS statistical program software (version 20 SPSS Inc., Chicago, IL).

\section{Results}

Clinical and biochemical characteristics of patients studied as whole sample or divided by presence/absence of renal impairment and/or prior cardiovascular event are reported in Table 1. Overall, the mean age was $68.3 \pm 10.6$ years, $74 \%$ were male, mean BMI $28.4 \pm 4.8 \mathrm{~kg} / \mathrm{m} 2$. At hospital admission BP was fairly controlled, mean systolic BP (SBP) was $133.8 \pm 15.9 \mathrm{mmHg}$ and mean DBP $75.7 \pm 9.5 \mathrm{mmHg}$. It is worth to note that the vast majority of patients (i.e. $89 \%$ ) were diagnosed as having arterial hypertension. As far as kidney function is concerned, mean eGFR was $72.1 \pm 22.3 \mathrm{ml} / \mathrm{min} / 1.73 \mathrm{~m}^{2}$ with $29 \%$ of patients showing eGFR $<60 \mathrm{ml} / \mathrm{min} / 1.73 \mathrm{~m} 2$, while median ACR was $1.4 \mathrm{mg} / \mathrm{mmol}$ (range 0.27 1471). One-hundred ninety-one patients (33\%) exhibited an increased ACR (including patients with either microalbuminuria (25\%) or macroalbuminuria (8\%). Two-hundred ninenty-two (42\%) patients had T2DM.

According to the purpose of the study, the whole population was then divided into 4 subgroups according to the presence/absence of eGFR $<60 \mathrm{ml} / \mathrm{min} / 1.73 \mathrm{~m} 2$ or prior CV event: eGFR $\geq 60 /$ no CV event (Group 
A), $n=306$ (43.7\%), eGFR $\geq 60 /$ yes CV event (Group B), $n=190$ (27.1\%), eGFR<60/no CV event (Group C), n=98 (14\%), eGFR<60/yes CV event (Group D), n=106 (15.2\%). Data reported in Table 1 show, as expected, some relevant differences among subgroups of patients. In fact, patients in group $D$ had the worst clinical and biochemical profile. They were older, with higher prevalence of diabetes, dyslipidemia and arterial hypertension $(p<0.01)$ for all. These patients also showed the highest values of ACR $(p<0.001)$ and the lowest values of eGFR $(p<0.01)$.

In Supplementary Table 1 and Table 2 we report clinical and biochemical features in further subgroups in which albuminuria or CKD replaced low eGFR.

Angiographic data

In Table 2 we report coronary angiographic data in the whole population and according to presence/absence of prior cardiovascular event or low eGFR. While 223 patients show no significant coronary lesions, 196 had three vessel disease. Figure 1 depicts the percentage of patients with 3VD distributed among the 4 subgroups of patients. Considering group $A$ as reference, the risk of having 3VD, was increased in group $B(O R=2.09 ; 95 \% \mathrm{Cl} 1.37-3.19)$, in group $\mathrm{C},(\mathrm{OR}=1.80 ; 95 \% \mathrm{Cl} 1.04-3.14)$ and, finally in group $D(O R=3.35 ; 95 \% \mathrm{Cl} 2.01-5.58)$ (Table $3 A)$. The risk carried by group $C$ was not significantly different from that carried by Group B: OR= 0.86; 95\% Cl 0.5-1.5 (Table 3B), showing a similar 3VD among these two groups of patients.

Results did not change when albuminuria or CKD replaced low eGFR in the different subgroups analysed (Supplementary Figure 1 and 2, Supplementary Table 3 and 4).

\section{Discussion}

In our study involving 700 patients undergoing CA, those with low eGFR and prior CV event carry the heaviest coronary atherosclerotic burden (i.e. percentage of patients affected by $3 \mathrm{VD}$ ). Low eGFR seems to give the same excess risk of prior $\mathrm{CV}$ event. At our knowledge, this is the first study comparing coronary atherosclerotic burden in this peculiar setting of patients.

Three-vessel disease is the most severe form of coronary atherosclerosis. In fact, therapeutic guidelines indicate these patients being at very high $\mathrm{CV}$ risk [11-13].

CKD is also associated with high risk of CHD events and mortality either in the general population [14] or in high-risk subjects such as those with known vascular disease [15-16], diabetes [17], hypertension [18], or CHD [19]. Natali et al. have clearly shown how mild reduction in GFR is associated with a more severe coronary artery disease [20].

While it is well known the causative relationship between augmented atherosclerotic burden with unstable, calcified plaques in patients with end stage renal disease scant information is available on the relationship between atherosclerotic burden with initial stages 3 CKD. More than a decade ago, Buzzelo et al. [21] demonstrated in an animal model of atherosclerosis with advanced kidney disease, the presence 
of large atherosclerotic plaque with unstable morphology rather than onset of new ones. Recently, Sugiyama et al have investigated coronary atherosclerotic plaque by optical coherence tomography. The authors confirmed with this sophisticate methodology that CKD is associated with the growth of lipidic plaques suggesting that the progression of CKD to more advanced stages might cause silent plaque rupture [22].

The American College of Cardiology/American Heart Association task force and the National Kidney Foundation fifteen years ago already recommended to consider CKD a coronary heart disease (CHD) risk equivalent [7]. The results of the Alberta Kidney Disease Network investigating a large population of more than one million of subjects confirmed the above indications stating that all-cause mortality is higher in patients with advanced CKD than those with a prior myocardial infarction [23]. Our results are in line with American College of Cardiology/American Heart Association task force and the National Kidney Foundation indications.

On the contrary, Wattanakit et al. have investigated the same issue analyzing 12,243 non diabetic subjects from the ARIC (Atherosclerosis Risk in Communities) study. They stratified the whole population by the presence/absence of CKD and myocardial infarction. After adjustment for several confounders, the authors found that CHD incidence and CVD mortality rates per 1,000 person-years were 4.1 and 1.0 among subjects with neither condition, 8.0 and 3.4 in CKD only, 18.8 and 7.0 in MI only, and 30.8 and 18.0 in $\mathrm{CKD}$ and $\mathrm{MI}$, thus concluding that stage $3 \mathrm{CKD}$ cannot be considered a CHD risk equivalent [24]. Although in our study we do not have information on cardiovascular events, the difference with our finding can be explained, at least partially, by the differences in the features of patients studied. In fact, Wattanakit et al. have investigated patients with prior MI and stage 3 CKD while in our study we have enrolled patients with a broad figure of cardiovascular disease and stage $\geq 3$ CKD. Moreover, patients with diabetes were excluded in the Wattanakit study.

Our study has some strength and weakness points. One major strength is the uni-centric nature of the study. In fact, all CA were performed and read by the same two co-authors (CV and NM). When the opinion was discordant, the judgment of a third independent person was asked. A second point is the method used to measure serum creatinine, calibrated to be traceable to an isotope dilution mass spectrometry (IDMS) IDMS-traceable. Among the limitations: first: the lack of information on hard cardiovascular events is relevant.

The second one: there is only one measurement of serum creatinine or albuminuria.

\section{Conclusions}

Our study states that patients with CKD and prior CV event have the worst atherosclerotic coronary burden. Patients with CKD carry a similar risk of coronary atherosclerotic burden of patients with prior CV event. Further larger prospective studies will clarify whether a similar coronary atherosclerotic burden will end up in similar cardiovascular outcomes. 


\section{Abbreviations}

CAD: Atherosclerotic coronary artery disease; CKD: Chronic kidney disease; eGFR: estimated glomerular filtration rate; $\mathrm{CHD}$ : coronary heart disease; $\mathrm{CA}$ : coronary angiography; $\mathrm{PCl}$ : percutaneous coronary intervention; BMI: Body mass index; BP: blood pressure; DBP: diastolic blood pressure; SBP: systolic BP; IDMS: isotope dilution mass spectrometry; ACR: urinary albumin creatinine ratio; HbA1c: Glycated haemoglobin; SVD: single vessel disease; 2 VD: two vessels disease; 3 VD: three vessels disease; T2DM: type 2 diabetes mellitus; ESKD: end stage kidney disease.

\section{Declarations}

Ethics approval and consent to participate: all procedures followed were in accordance with the ethical standards of the responsible committee on human experimentation. The study was approved by local Ethical Committee of Scientific Institute "Casa Sollievo della Sofferenza", San Giovanni Rotondo, Foggia. Informed prior consent was obtained in written format from each subject.

Consent for publication: not applicable.

Availability of data and materials: all data generated or analysed during this study are included on this published article [and its supplementary information files].

Competing interests: the authors declare that they have no competing interests.

Funding: this research was funded by "AMD", Associazione Medici Diabetologi.

Authors' Contributions: Conception and design of total study, SD and PP; Acquisition of data, PP, AMa, NM, EVG, MMD, VM, AM, APP, GV, AR, CV; Analysis and interpretation of data, PP, SD, RP; Drafting of manuscript and critical revision, all authors. All authors have read and approved the manuscript.

Acknowledgements: We thank Mrs. Caterina Mirijello for writing assistance.

\section{References}

1 GBD 2013. Mortality and Causes of Death Collaborators, Global, regional, and national age-sex specific all-cause and cause-specific mortality for 240 causes of death, 1990-2013: a systematic analysis for the Global Burden of Disease Study 2013. Lancet. 2015; 385:117-171.

2 Pekkanen J, Linn S, Heiss G, Suchindran CM, Leon A, et al. Ten-year mortality from cardiovascular disease in relation to cholesterol level among men with and without preexisting cardiovascular disease. $\mathrm{N}$ Engl J Med. 1990;322:1700-7.

3 Brønnum-Hansen H, Jørgensen T, Davidsen M, Madsen M, Osler M, Gerdes LU, et al. Survival and cause of death after myocardial infarction: the Danish MONICA study. J Clin Epidemiol. 2001;54:1244-50. 
4 Brück K, Stel VS, Gambaro G, Hallan S, Völzke H, Ärnlöv J,et al.; European CKD Burden Consortium. CKD prevalence varies across the European general population. J Am Soc Nephrol. 2016;27:2135-47.

5 Reinecke H, Trey T, Matzkies F, Fobker M, Breithardt G, Schaefer RM. Grade of chronic renal failure, and acute and long-term outcome after percutaneous coronary interventions. Kidney Int. 2003;63:696-701.

6 Go AS, Chertow GM, Fan D, McCulloch CE, Hsu CY. Chronic kidney disease and the risks of death, cardiovascular events, and hospitalization. N Engl J Med. 2004;351:1296-305.

7 Sarnak MJ, Levey AS, Schoolwerth AC, Coresh J, Culleton B, Hamm LL, et al. Kidney disease as a risk factor for development of cardiovascular disease: a statement from the American Heart Association councils on kidney in cardiovascular disease, high blood pressure research, clinical cardiology, and epidemiology and prevention. Circulation;108:2154-69.

8 Thompson S, James M, Wiebe N, Hemmelgarn B, Manns B, Klarenbach S, et al.; Alberta Kidney Disease Network. Cause of death in patients with reduced kidney function. J Am Soc Nephrol. 2015;26:2504-11.

9 Kunimura A, Ishii H, Uetani T, Harada K, Kataoka T, Takeshita M, et al. Prognostic Value of Albuminuria on Cardiovascular Outcomes After Elective Percutaneous Coronary Intervention. Am J Cardiol. 2016;117:714-9.

10 D'Errico MM, Mangiacotti A, Graziano D, Massa V, Piscitelli P, Vendemiale G, et al. Kidney disease measures are associated with the burden of coronary atherosclerosis, independently of diabetes. Acta Diabetol. 2017; 54:1065-1068.

11 Lopes NH, Paulitsch Fda S, Gois AF, Pereira AC, Stolf NA, Dallan LO, et al. Impact of number of vessels disease on outcome of patients with stable coronary artery disease: 5-year follow-up of the Medical, Angioplasty, and bypass Surgery study (MASS). Eur J Cardiothorac Surg 2008; 33:349-354.

12 Lawrie GM, Morris GC Jr, Calhoon JH, Safi H, Zamora JL, Beltengady M, et al. Clinical results of coronary bypass in 500 patients at least 10 years after operation. Circulation. 1982;66:11-5.

13 van Domburg RT, Kappetein AP, Bogers AJ. The clinical outcome after coronary bypass surgery: a 30year follow-up study. Eur Heart J. 2009;30(4):453-8.

14 Fox CS, Matsushita K, Woodward M, Bilo HJ, Chalmers J, Heerspink HJ, et al.; Chronic Kidney Disease Prognosis Consortium. Associations of kidney disease measures with mortality and end-stage renal disease in individuals with and without diabetes: a meta-analysis. Lancet. 2012;380:1662-73.

15 Weiner DE, Tighiouart H, Stark PC, Amin MG, MacLeod B, Griffith JL, et al. Kidney disease as a risk factor for recurrent cardiovascular disease and mortality. Am J Kidney Dis. 2004;44:198-206. 
16 van der Velde M, Matsushita K, Coresh J, Astor BC, Woodward M, Levey A, et al. Lower estimated glomerular filtration rate and higher albuminuria are associated with all-cause and cardiovascular mortality. A collaborative meta-analysis of high-risk population cohorts. Kidney Int. 2011;79:1341-52.

17 Mann JF, Gerstein HC, Pogue J, Bosch J, Yusuf S. Renal insufficiency as a predictor of cardiovascular outcomes and the impact of ramipril: the HOPE randomized trial. Ann Intern Med. 2001;134:629-36.

18 Shulman NB, Ford CE, Hall WD, Blaufox MD, Simon D, Langford HG, et al. Prognostic value of serum creatinine and effect of treatment of hypertension on renal function. Results from the hypertension detection and follow-up program. The Hypertension Detection and Follow-up Program Cooperative Group. Hypertension. 1989;13:I80-93.

19 Walsh CR, O'Donnell CJ, Camargo CA Jr., Giugliano RP, LloydJones DM. Elevated serum creatinine is associated with 1-year mortality after acute myocardial infarction. Am Heart J. 2002;144:1003-11.

20 Natali A, Boldrini B, Baldi S, Rossi M, Landi P, Severi S, et al. Impact of mild to moderate reductions of glomerular filtration rate on coronary artery disease severity. Nutr Metab Cardiovasc Dis. 2014;24:681-8

21 Buzello M, Tornig J, Faulhaber J, Ehmke H, Ritz E, Amann K. The apolipoprotein e knockout mouse: a model documenting accelerated atherogenesis in uremia. J Am Soc Nephrol. 2003;14:311-6.

22 Sugiyama T, Kimura S, Ohtani H, Yamakami Y, Kojima K, Sagawa Y, et al. Impact of chronic kidney disease stages on atherosclerotic plaque components on optical coherence tomography in patients with coronary artery disease. Cardiovasc Interv Ther. 2017;32:216-224.

23 Tonelli M, Muntner P, Lloyd A, Manns BJ, Klarenbach S, Pannu N, et al; Alberta Kidney Disease Network. Risk of coronary events in people with chronic kidney disease compared with those with diabetes: a population-level cohort study. Lancet. 2012;380:807-14.

24 Wattanakit K, Coresh J, Muntner P, Marsh J, Folsom AR. Cardiovascular risk among adults with chronic kidney disease, with or without prior myocardial infarction. J Am Coll Cardiol. 2006;48:1183-9.

\section{Tables}

Table 1. Characteristics of 700 patients studied who underwent to CA; whole population and subgroups divided according to the presence/absence of eGFR $<60 \mathrm{ml} / \mathrm{min} / 1.73 \mathrm{~m}^{2}$ and the presence/absence of previous cardiovascular event. 


\begin{tabular}{|c|c|c|c|c|c|c|}
\hline & $\begin{array}{l}\text { Whole } \\
\text { population }\end{array}$ & $\begin{array}{l}\text { eGFR } \geq 60 \\
\text { no event } \\
\text { (Group A) }\end{array}$ & $\begin{array}{l}\text { eGFR } \geq 60 \\
\text { yes event } \\
\text { (Group B) }\end{array}$ & $\begin{array}{c}\text { eGFR<60 } \\
\text { no event } \\
\text { (Group C) }\end{array}$ & $\begin{array}{l}\text { eGFR }<60 \\
\text { yes event } \\
\text { (Group D) }\end{array}$ & $\begin{array}{c}\mathbf{p} \\
\text { value }\end{array}$ \\
\hline & $\mathrm{n}=700$ & $\mathrm{n}=306$ & $\mathrm{n}=190$ & $\mathrm{n}=98$ & $\mathrm{n}=106$ & \\
\hline Male n (\%) & $521(74 \%)$ & $231(75.5 \%)$ & $163(85.8 \%)$ & $58(59.2 \%)$ & $69(65.1 \%)$ & $<0.01$ \\
\hline Age (years) & $68.3 \pm 10.6$ & $65.1 \pm 10.6$ & $66.9 \pm 9.2$ & $74.2 \pm 10.2$ & $74.5 \pm 7.9$ & $<0.01$ \\
\hline BMI $\left(\mathrm{Kg} / \mathrm{m}^{2}\right)$ & $28.4 \pm 4.8$ & $28.2 \pm 4.8$ & $28.3 \pm 4.2$ & $28.5 \pm 4.9$ & $28.9 \pm 5.9$ & 0.652 \\
\hline SBP (mmHg) & $133.8 \pm 15.9$ & $133.1 \pm 15.4$ & $134.5 \pm 15.5$ & $134.6 \pm 17.5$ & $133.9 \pm 16.8$ & 0.773 \\
\hline DBP (mmHg) & $75.7 \pm 9.5$ & $75.8 \pm 9.4$ & $75.3 \pm 8.8$ & $77 \pm 10.9$ & $75.3 \pm 9.9$ & 0.545 \\
\hline Triglycerides $(\mathrm{mg} / \mathrm{dL})^{\circ}$ & $\begin{array}{c}142(33- \\
855)\end{array}$ & $\begin{array}{c}134(33- \\
855)\end{array}$ & $\begin{array}{c}146(52- \\
788)\end{array}$ & $\begin{array}{c}127(40- \\
440)\end{array}$ & $\begin{array}{c}156(51- \\
485)\end{array}$ & 0.029 \\
\hline HDL-c (mg/dL) & $46.2 \pm 13.3$ & $47.4 \pm 12.9$ & $45.7 \pm 12.7$ & $46.6 \pm 15$ & $43.4 \pm 13.5$ & 0.073 \\
\hline LDL-c (mg/dL) & $95.5 \pm 35.1$ & $110.4 \pm 35.5$ & $86.7 \pm 30.5$ & $93.2 \pm 34.5$ & $91.7 \pm 32.3$ & $<0.01$ \\
\hline eGFR $\left(\mathrm{mL} / \mathrm{min} / 1.73 \mathrm{~m}^{2}\right)$ & $72.1 \pm 22.3$ & $84.5 \pm 13.9$ & $81.8 \pm 13.3$ & $45.8 \pm 12.7$ & $42.9 \pm 13$ & $<0.01$ \\
\hline ACR (mg/mmol) ${ }^{\circ}$ & $\begin{array}{l}1.4(0.27- \\
1471)\end{array}$ & $\begin{array}{l}1.2(0.34- \\
141.4)\end{array}$ & $\begin{array}{l}1.2(0.36- \\
831.01)\end{array}$ & $\begin{array}{l}1.9(0.41- \\
780.9)\end{array}$ & $\begin{array}{l}3.3(0.27- \\
1471)\end{array}$ & $<0.001$ \\
\hline Normoalbuminuria $\mathrm{n}(\%)$ & $392(67 \%)$ & $\begin{array}{c}189 \\
(77.1 \%) \\
\end{array}$ & 116 (69\%) & 45 (56.3\%) & $42(45.2 \%)$ & - \\
\hline Microalbuminuria n (\%) & 149 (25\%) & $49(20 \%)$ & $44(26.2 \%)$ & 26 (32.5\%) & $30(32.3 \%)$ & - \\
\hline Macroalbuminuria n (\%) & $45(8 \%)$ & $7(2.9 \%)$ & $8(4.8 \%)$ & $9(11.3 \%)$ & $21(22.6 \%)$ & $<0.01$ \\
\hline Smokers n (\%) & $152(22 \%)$ & 78 (25.5\%) & 49 (25.8\%) & $14(14.3 \%)$ & $11(10.4 \%)$ & 0.012 \\
\hline Dyslipidemia n (\%) & $515(74 \%)$ & $190(62.1 \%)$ & $166(87.4 \%)$ & $64(65.3 \%)$ & $95(89.6 \%)$ & $<0.01$ \\
\hline $\begin{array}{l}\text { Lipid-lowering treatment with } \\
\text { Statins/Fibrates n (\%) }\end{array}$ & $492(70 \%)$ & 178 (58.2\%) & 160 (84.2\%) & 60 (61.2\%) & 94 (88.7\%) & $<0.01$ \\
\hline Arterial hypertension n (\%) & $626(89 \%)$ & $251(82 \%)$ & $174(91.6 \%)$ & 95 (96.9\%) & $106(100 \%)$ & $<0.01$ \\
\hline Use of ACE-Is/ARBs n (\%) & $498(71 \%)$ & $204(66.7 \%)$ & $136(71.6 \%)$ & $74(75.5 \%)$ & $84(79.2 \%)$ & 0.063 \\
\hline T2DM n (\%) & $292(41.7 \%)$ & 97 (31.7\%) & $81(42.6 \%)$ & $50(51.0 \%)$ & $64(60.4 \%)$ & $<0.01$ \\
\hline
\end{tabular}

Mean \pm SD, absolute frequency (percentage) and ${ }^{\circ}$ median (range). BMI, body mass index; SBP, systolic blood pressure; DBP, diastolic blood pressure; HDL, high-density lipoprotein cholesterol; LDL, low-density lipoprotein cholesterol; eGFR, estimated glomerular filtration rate; ACR, urinary albumin-to-creatinine ratio; ACE-Is, angiotensin converting enzyme-inhibitors; ARBs, angiotensin II receptor antagonists; T2DM, type 2 diabetes mellitus. Patient's baseline missing data: ACR in 114 patients; CKD in 83 patients.

Table 2. Angiographic reports of severity of coronary artery disease among patients with or without renal impairment and previous cardiovascular event. 


\begin{tabular}{|c|c|c|c|c|c|}
\hline $\mathrm{CAD}$ & Whole population & Group A & Group B & Group C & Group D \\
\hline No-vessel disease (No-VD) & $223(32 \%)$ & 133 (44\%) & $\mid 36(19 \%)$ & $35(36 \%)$ & $19(18 \%)$ \\
\hline Single-vessel disease (S-VD) & $152(22 \%)$ & $67(22 \%)$ & 47 (25\%) & 19 (19\%) & $19(18 \%)$ \\
\hline Two-vessel disease (2-VD) & 129 (18\%) & $50(16 \%)$ & $43(22 \%)$ & $15(15 \%)$ & $21(20 \%)$ \\
\hline Three-vessel disease (3-VD) & $196(28 \%)$ & $56(18 \%)$ & $64(34 \%)$ & $29(30 \%)$ & $47(44 \%)$ \\
\hline
\end{tabular}

* Adjusted by age and gender

$\mathrm{p}=<0.01$

CAD, Atherosclerotic coronary artery disease; Group A, eGFR $\geq 60 /$ no event; Group B, eGFR $\geq 60 /$ yes event; Group C, eGFR<60/no event; Group D, eGFR<60/yes event.

Table 3A. Risk for having three-vessel coronary disease according to presence/absence of eGFR $<60$ or prior cardiovascular events, considering group A as reference.

\begin{tabular}{|c|c|c|c|}
\hline Group A & Group B & Group C & Group D \\
Reference & OR, 95\%CI: 2.09 (1.37-3.19) & OR, 95\%CI: 1.80 (1.04-3.14) & OR, 95\%CI: 3.35 (2.01-5.58) \\
& & & \\
\hline
\end{tabular}

ORs are adjusted by age and gender.

Table 3B. Risk for having three-vessel coronary disease according to presence/absence of eGFR $<60$ or prior cardiovascular events: comparison between groups.

\begin{tabular}{|c|c|c|c|}
\hline Group A & Group B & Group C & Group D \\
& OR, 95\%CI: 2.09 (1.37-3.19) & OR, 95\%CI: 0.86 (0.49-1.51) & OR, 95\%CI: 1.85 (1.03-3.34) \\
& & & \\
\hline
\end{tabular}

ORs are adjusted by age and gender.

OR, odds ratio; 95\%CI, 95\% confidence of intervals; eGFR, estimated glomerular filtration rate; Group A, eGFR $\geq 60$ /no event; Group B, eGFR $\geq 60$ /yes event; Group C, eGFR<60/no event; Group D, eGFR<60/yes event. 


\section{Figures}

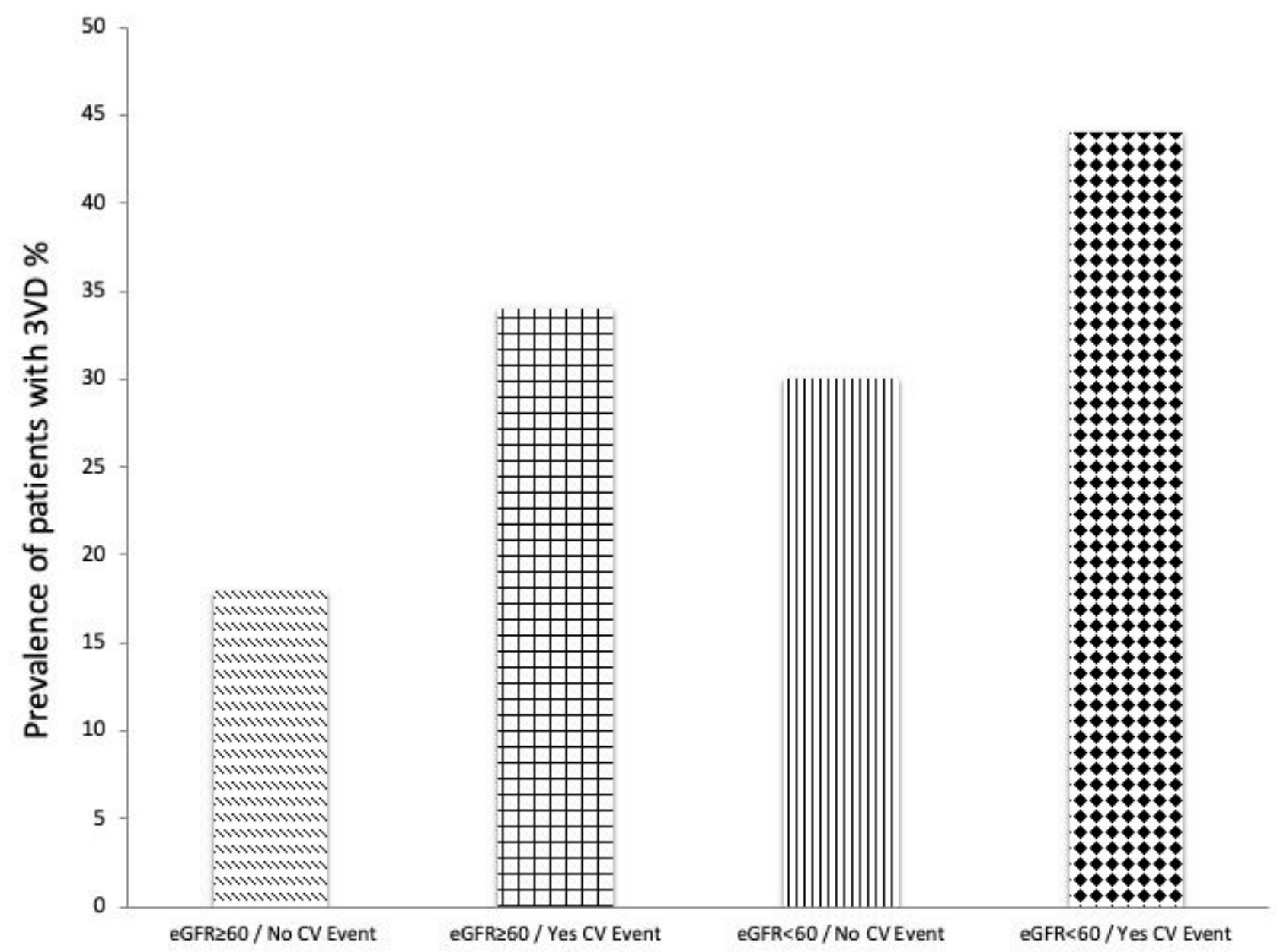

Figure 1

Figure 1

Percentage of patients with 3VD distribution in 4 subgroups of patients, according to the presence/absence of eGFR $<60 \mathrm{ml} / \mathrm{min} / 1.73 \mathrm{~m} 2$ or prior cardiovascular event.

\section{Supplementary Files}

This is a list of supplementary files associated with this preprint. Click to download.

- AdditionalFigure2.jpeg

- AdditionalTable1.docx 
- AdditionalTable2.docx

- AdditionalTable4.docx

- AdditionalFigure1.jpeg

- AdditionalTable3.docx 